\title{
MAX: A Method for Evaluating the Post-use User eXperience through Cards and a Board
}

\author{
Emanuelle Cavalcante ${ }^{1,2}$, Luis Rivero ${ }^{1}$ and Tayana Conte $^{1}$ \\ 1USES Research Group, Instituto de Computação, Universidade Federal do Amazonas (UFAM) \\ Manaus, AM - Brazil \\ ${ }^{2}$ FPF Tech, Manaus, AM - Brazil \\ emanuelle.cavalcante@fpf.br, \{luisrivero,tayana\}@icomp.ufam.edu.br
}

\begin{abstract}
User Experience (UX) is one of the most important attributes for the success and quality of a software product. UX explores how a person uses an application, and the emotional and behavioral consequences of such use. Although several UX evaluation methods allow gathering information on the reasons for a poor UX, some of them tend to make users feel uncomfortable, such as asking direct questions to shy users. This paper presents our proposal for evaluating UX, the Method for the Assessment of eXperience (MAX), which through cards and a board intends to motivate users to report their experience. The MAX method does not require experienced evaluators for performing the evaluation. Instead, this method is intended at software engineers willing to obtain data on UX and make users feel comfortable during the evaluation. To verify the feasibility of the MAX method from the point of view of users, we conducted a pilot study. The results showed that the MAX method has proven useful for evaluating the $\mathrm{UX}$ of finished or prototyped software applications. Also, we have made improvements in the method to meet users' needs when reporting their experience, and gathering data on factors affecting the UX.
\end{abstract}

Keywords: User eXperience; Evaluation Method; Software Quality.

\section{INTRODUCTION}

Usability has been considered one of the main attributes that represent quality in an instrumental, task-oriented view of interactive products [1]. However, despite the increasing attention that usability has received in the development of software applications [2], a new term, "User eXperience" (UX), has emerged as an umbrella phrase for new ways of understanding and studying the quality in use of interactive products [1].

UX is an important attribute for the success of software products, searching for new approaches for their design and evaluation, which accommodates experiential qualities of technology use rather than product qualities [3]. According to the ISO 9241 [4], user experience is defined as "a person's perceptions and responses that result from the use or anticipated use of a product, system or service”. Such definition is complemented by the definitions of other authors. For instance, Law et al. [5] state that UX explores how users feel about the use of a product, or in other words, the emotional and affective aspects. Thus, UX turns essential in order for a software to be accepted by its users since, besides being usable and work correctly, the software should be emotionally appealing [6].

The increasing interest in the improvement of UX has been the motivation for the creation of new evaluation methods that allow capturing the users' emotions and the aspects that affect the reported emotions [7]. In that context, it is necessary to highlight the difference between usability evaluation methods and UX evaluation methods. Usability tests tend to focus on task performance, while UX focuses on the experience that was lived by the user and his/her emotions.

As it is subjective, UX deals with the feelings and thoughts of an individual regarding the use of a software, product or service. According to Law et al. [5], usability measures such as execution time or number of errors are not enough to measure the user experience of a product. Also, it is not always easy for a user to realize what (s)he is feeling or even express his/her user experience. Therefore, it is necessary to employ new approaches that stimulate and guide the user during the report of his/her experience. The report of the use by the user allows the evaluator to gain insights on the experienced problems and also understand positive aspects of the product use. Furthermore, it is possible to gather data regarding if the product was accepted by the user or not.

Vermeeren et al. [7] state that there is a high number of UX evaluation methods being employed by both the industry and the academy. Nonetheless, according to Miles et al. [8], some methods can cause discomfort to the users who participate from the evaluation since it forces them to report their experience. Users can feel forced to participate in the evaluation sessions and this can cause bias in the report of their experience [6]. Consequently, it is necessary to develop practical methods that are easy to use and do not feel like a chore, so the users can be confortable when reporting their experience [7]. Also, these methods must be easy to use from the point of view of software engineers, so practitioners from the software industry are able to apply them and gather data to improve the quality of software applications under development [7].

In order to propose a UX evaluation method that is easy to use and motivates users to report their experience, we have proposed the Method for the Assessment of eXperience (MAX). MAX introduces a set of cards and a board to guide the user through the UX evaluation process. That way, the 
evaluator can gather information on the user's emotions, how easy and useful it was to use the system, and his/her intention to use the system again if given the chance. The MAX method can be applied at any stage of the software development process, after the use of mockups, prototypes, or the final versions of interactive systems.

This paper presents the initial version of the MAX method, and its initial evaluation from the point of view of users through a pilot study. We evaluated MAX through questionnaires in order to gather information regarding if it was easy to apply by the users. Based on the results from the evaluation, we have made improvements in MAX, generating a second version. As a result of this application of the MAX method, we were able to evaluate its feasibility for the evaluation of UX.

This paper is organized as follows. Section 2 presents the background and related work of this research, where we provide UX definitions and a brief description of some of the proposed UX evaluation methods. Next, Section 3 describes the proposed MAX method, while Section 4 describes the execution and results from the pilot study and the initial improvements over MAX. Finally, Section 5 presents our conclusions and future work regarding this research.

\section{BACKGROUND AND RELATED WORK}

User eXperience involves all aspects of the user interaction and aims at guaranteeing that software systems become satisfying, interesting, useful, motivating, beautiful and adequate [9]. According to Roto [10], such experience includes the emotions, preferences, physical and psychological reactions of the user that can occur: (a) before usage, or in other words, the expectations of the user regarding the software product; (b) during usage, which is the momentary experience of the user; and (c) after usage (post-use), when it is possible to verify if the users' expectations were actually met. Furthermore, UX is a consequence of the internal state of the user (his/her expectations, needs, motivations, humor, and others), the features of the system (utility, ease of use, functionality, and others) and the context and environment in which the interaction between the user and the system occurs [11].

UX evaluation plays an important role in the development of interactive applications, since it assesses their value regarding how the users will apply, perceive, and learn the software, as well as how it will evolve and adapt to the users' changing expectations [12]. In that context, UX evaluation methods can be employed to gauge the product success in the real market and attract potential customers [13].

In order to identify which UX evaluation methods were proposed, Vermeeren et al. [7] carried out a review. Such review allowed identifying 96 UX evaluation methods that can be applied in different types of applications (desktop, Web, mobile, and others) and in different phases of the software development process (analysis, design, test, coding, and others). From the set of identified UX methods for evaluating the after usage of a system, one can name: scales, online surveys and probes. We will detail these methods and their (dis)advantages as follows.
Scales such as the Self-Assessment Manikin (SAM) [14] and the Unified Theory of Acceptance and Use of Technology (UTAUT) [15] allow evaluating the opinion of the users by measuring specific attributes related to UX. Through the SAM scale, it is possible to evaluate three dimensions: (a) Pleasure (pleasure/displeasure), (b) Dominance (in control of the situation/controlled by the situation), and (c) Arousal (calmed/excited). UTAUT, on the other hand, focuses on aspects related to technology acceptance, measuring effort, performance and facilitator conditions. Although scales demand less time to be employed and shy users may feel more comfortable when applying these methods, the collected data may not provide insights on the causes for a poor UX.

Online surveys can also be employed to gather information on UX. An example is the AttrakDiff [16], which allows several users from different locations and profiles to provide information on pragmatic and hedonic attributes of a product. The main issue with collecting data online is that the evaluator does not have control over the proper filling of the questionnaire, and cannot return to the users for further feedback.

Probes are another alternative for evaluating UX. With probes, users can be encouraged to report their experience. For instance, the Emocards [17], which are 16 drawings of faces (8 male and 8 female) illustrate different emotional responses to an evaluated product. When a user picks one (or more) of the emocards, (s)he can report his/her experience explaining the reasons that motivated him/her to choose it. However, the user may explain what (s)he thinks is important to be explained, concealing relevant information from the evaluators.

The methods cited above demand low costs in their application and are easy to use from the point of view of users or evaluators [7]. However, there are some disadvantages that can influence the results of the UX evaluation. According to Tähti and Niemelä [6], users applying methods that employ the representation of emotions may not be able to relate their emotions with the drawings for not understanding the representations. Also, Miles et al. [8] state that the majority of the UX evaluation methods might be intrusive, and that the fact of asking direct questions to the users about their emotions might make them feel uncomfortable. This can have a negative effect on the results of the UX evaluation, since users can hide vital information or express different emotions than the ones they are really feeling in order to please the evaluators. Finally, as mentioned before, some scales and forms may not provide information on the causes for choosing a specific answer, and not being able to request further information on why the user chose a specific answer may conceal relevant information for improving the UX. Considering the disadvantages of the current UX evaluation methods, it is necessary to provide new methods that meet the needs of the evaluators, make users feel comfortable, and make it easier for them to report their emotions. In order to meet these goals, we proposed MAX.

\section{THE METHOD FOR AssESSING EXPERIENCE (MAX)}

The Method for the Assessment of eXperience (MAX) is a post-use method that aims at evaluating the general experience 
of a user regarding an interactive application. MAX can be employed after the use of mockups, prototypes, interactive systems, or any artifact that allows user interaction.

The evaluation is performed through the use of cards and a board. The MAX cards allow evaluating the UX in terms of four categories: (a) Emotion, (b) Ease of Use, (c) Usefulness and (d) Intention to Use. These categories are similar to the evaluated aspects in technology acceptance methods, which consider usefulness, ease of use and intention [15]. As emotions are inseparable from cognition and are part of a user judgment about a system [18], we have also considered them in our method. In that context, the Emotion category focuses on the importance of the emotional aspects, since the experience considers the emotions, preferences and psychological reactions of the user [10]. To create the cards from the Emotion category, we considered the wheel of emotions by Plutchik [19]. The other three categories (Ease of Use, Usefulness and Intention to Use) were considered as they describe the necessary elements for achieving a positive UX [1]. In that context, the Ease of Use category aims at evaluating usability aspects of the application, while the Usefulness category aims at evaluating the user perception regarding how much the application contributes to the execution of his/her tasks. Finally, the Intention of Use category evaluates if the user would use or recommend the application.

Each MAX card presents an avatar to portray and express the possible reactions that a user can express regarding the evaluated system. Fig. 1 presents the avatar that we developed for one of the items of the Emotion category. We designed the avatar as a human cartoon form in order for the user to create empathy with the cards and be able to express him/herself more easily. Also, we chose to design cards that would not appear too formal, so users would not think of the evaluation as a chore, and would see it as something entertaining and comfortable to do.

The MAX deck of cards is inspired in a conventional deck of cards, where each evaluated UX category is represented by a symbol and color. Also, to allow users to express the intensity of an emotion, each card has an associated scale. These scales were developed and added to each of the items from the MAX categories in order to create the cards. Fig 1 also shows an example of the applied scales to describe the intensity of two items from the emotion category (a) Happy, positive and (b) Sad, negative. The cards that evaluate the UX in a positive way present a green scale, while the cards evaluating the UX in a negative way present red scales.

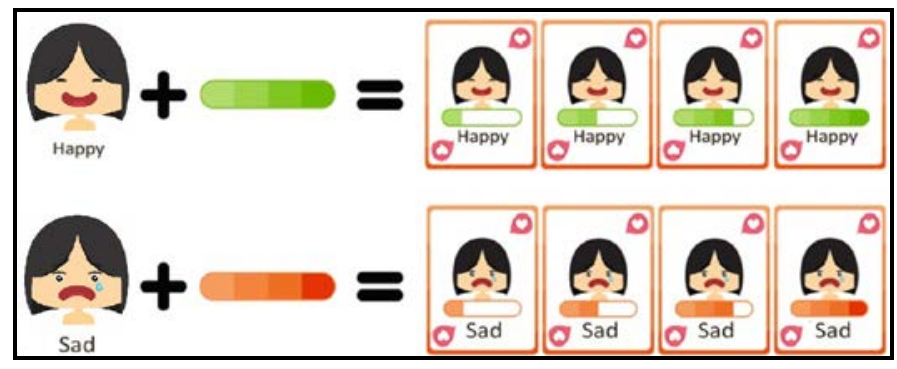

Figure 1. Avatar and intensity scales from the MAX method (v1)
Fig. 2 presents the evaluated items for each of the categories of the first version (v1) of the MAX method. At all, the MAX v1 provided 92 cards (considering all the items and their possible intensities): (a) 40 for the Emotion category, (b) 20 for the Intention to Use category, (c) 16 for the Utility category, and 16 for the Ease of Use category.

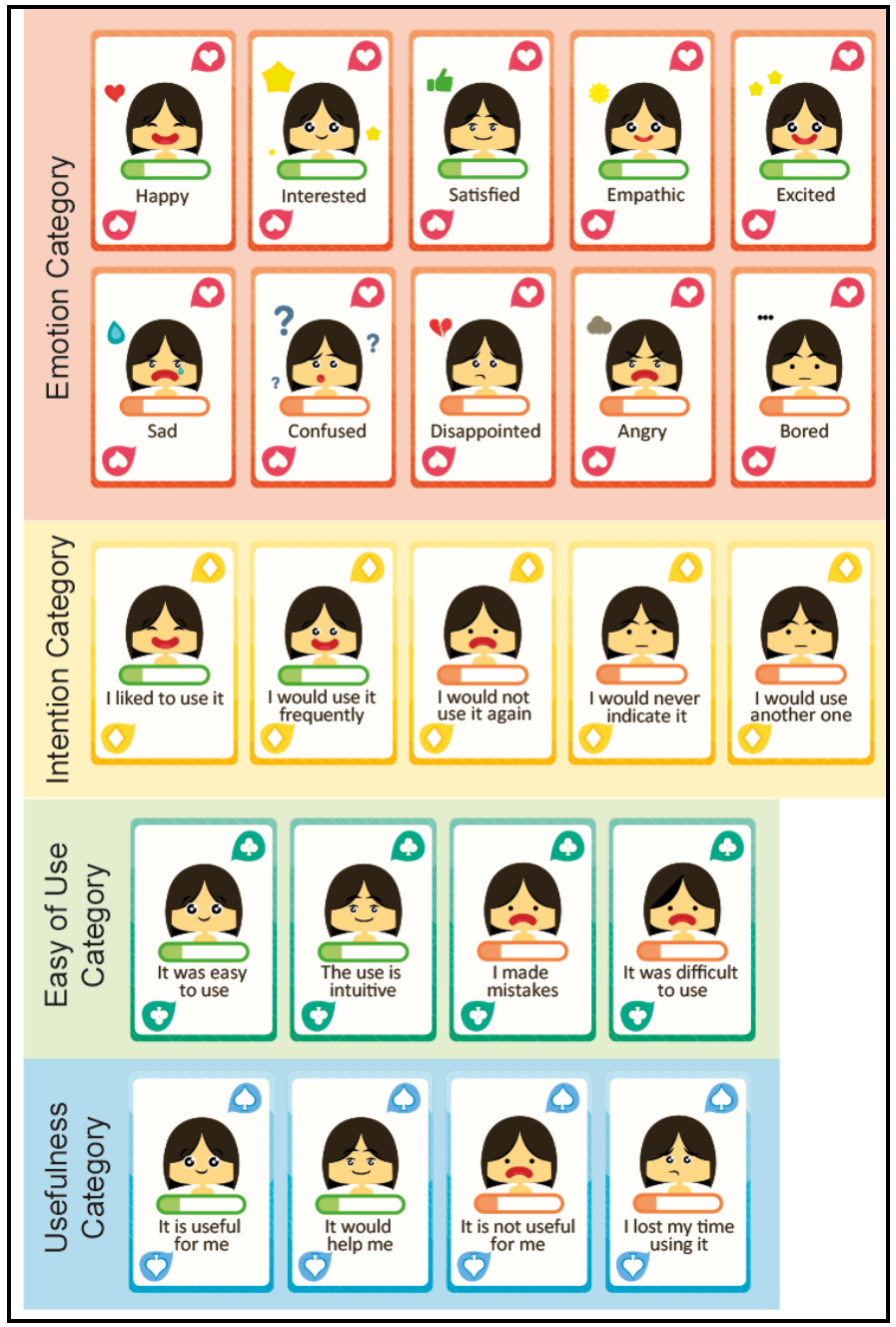

Figure 2. Evaluated items from the MAX method (v1)

In order to allow a much more dynamic application of the cards, we also developed a board. Such board shows which questions the user must answer when selecting the cards for reporting his/her experience. Initially, the board presented four questions, one for each of the evaluated categories within the MAX method: (a) What did you feel when using it? (Emotion category), (b) Was it easy to use? (Ease of Use category), (c) Do you wish to use it? (Intention category), and (d) Was it useful? (Usefulness category). Fig. 3 shows the MAX board for the first version of the MAX method.

In order to motivate users to choose at least two cards, we also added slots for each of the categories within the MAX method. These slots are spaces in which the user can place the MAX cards. We added these slots because a single card does not provide enough information regarding the user experience. 
Two or more cards, on the other hand, create a sequence which makes the report clearer for the evaluator.

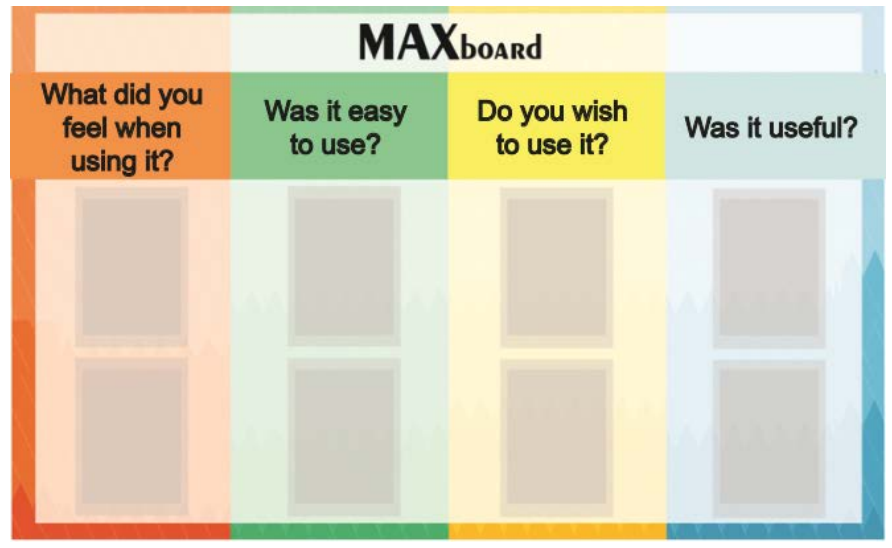

Figure 3. MAX Board (v1)

The application process of the MAX method is illustrated in Fig. 4. First the user must experience the software by carrying out tasks using a mockup, prototype of finished application (see Fig. 4 stage A). Then, the moderator presents the MAX method and the user must choose and place the cards in the board in order to report his/her experience (see Fig. 4 stage B). In this stage, the user is also allowed to express his/her opinions orally, explaining why (s)he chose a specific card. Finally, the evaluator collects and records the user's choice of cards and checks the occurrences of the cards associated with the reason for choosing them. It is important that the user is encouraged to talk about the choice of card as this information will help the evaluator to recognize what affects the user experience (see Fig. 4 stage C). Finally, the UX evaluator must generate a report listing the selected cards or if (s)he wishes, a picture of the board can be taken as a record of the evaluation.

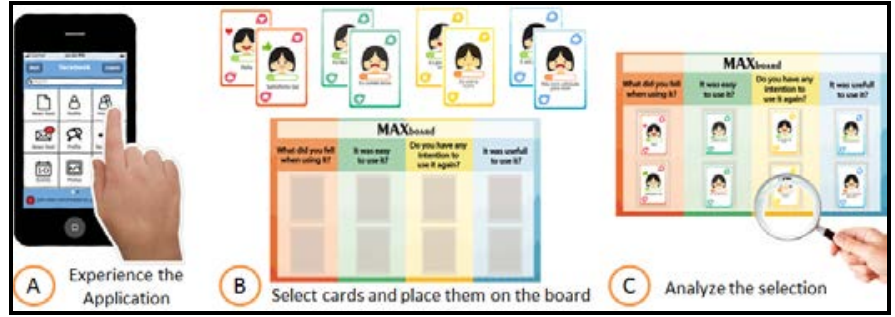

Figure 4. Application process of the MAX method: (a) Experience the Application; (b) Select cards and place them on the board; and (c) Analyze the selection.

\section{PILOT STUdY AND INITIAL IMPROVEMENTS ON THE MAX METHOD}

As cited by Isomursu et al. [20], UX evaluation methods need to be to provide useful information to evaluators and positive experiences to users applying them. Thus, to evaluate the feasibility of the MAX method, verifying if it could be employed in the post-use UX evaluation of a system by users, we carried out a pilot study. This study aimed at assessing the opinion of users regarding the use of the method, evaluating if they felt comfortable when reporting their experience.
We carried out this study with three potential users of a Web application for a telecommunications company (which is referred to as ALFA to retain anonymity). The users signed a consent form before starting the study. The consent form explained the goals of the study and its activities, the anonymity of the subjects' data, and that the study was safe as users would only have to experience an application and provide feedback. Users were free to (dis)agree with participating in the study. The study had three stages: (a) testing the system, in which the users tried the application; (b) UX evaluation, in which the users carried the post-use evaluation using the MAX method; and (c) feedback, in which the users answered a questionnaire regarding their opinion towards the MAX method. Before carrying out the study, we prepared the following materials: (a) a consent form (explained above); (b) the MAX cards and a sketch of the MAX board (see Section 3); (c) a scenario describing the goals that the user had to accomplish in the evaluated system; and (d) the feedback questionnaire.

In the execution of the study, we invited the users to participate in the evaluation of the Web application of the ALFA company. The users were chosen by convenience and they tried to carry out the following tasks: access a detailed bill and print it. Then, the users employed the MAX method. We highlight that the there was no need for training any software engineer in using the method. Since we aimed at evaluating MAX from the point of view of users, it was more suitable that an experience evaluator (one of the authors of the method) tested it with the users. Also, the users knew that they were able to end the evaluation session whenever they wanted.

At the end of the tests we took pictures of the chosen cards. Fig. 5 shows two different selections from two different types of users. The first selection of cards (Part A) was positive due to the experience of the user with the application. This user stated that (s)he was a client of ALFA and therefore, knew how to use the application. As (s)he managed to carry out the tasks, this user had a positive experience, indicating that (s)he felt satisfied and interested. Also, this user indicated that the application was intuitive and easy to use, and that (s)he would to use it again, because (s)he liked it. However, users experiencing the application for the first time did not have a positive experience. During the application of the MAX method, we identified the following problems regarding the system according to the users' reports: (a) the users had difficulty in requesting a password due to the unclear solicitation process; (b) the system feedback took time; and (c) the users had difficulties in finding the detailed bill, as they only found the paying value with no details on their service consumption. These problems negatively affected the UX of the subject whose cards selection is shown in Fig. 5 Part B. As we can see, this user felt satisfied but confused. Also, (s)he stated that the application was easy to use and intuitive, but not that much. And most importantly, that although the system could be useful and helpful, (s)he would not use it, or would use another system if available.

To assess the opinion of users towards the MAX method, users were asked to fill out a feedback questionnaire with the following questions: (a) Does the method feel intuitive and easy to use? and (b) Is the method and the instruments related 
to it easy to use, learn and understand?. These questions were considered, as they have been employed by other authors evaluating the ease of use of UX evaluation methods from the point of view of user [20]. Also, we asked the subjects to indicate if they would use MAX again. Overall, the answers to the questionnaires were positive. For instance, the users indicated that the board helped them when selecting the cards due to its guide questions. Also, the users stated that the MAX cards were useful since the avatar was clear and they managed to associate the depicted emotion with the label on the card.

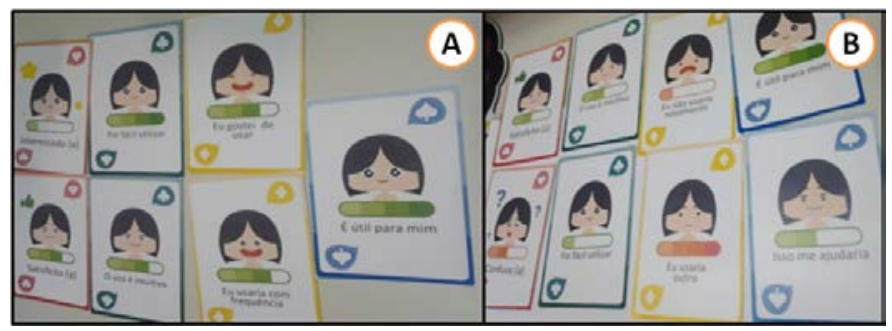

Figure 5. Selection of the MAX cards: (A) Positive and (B) Negative.

When describing their difficulties with the method, the users reported the high number of cards. Also, another user indicated that neutral cards were missing. This user stated that neutral cards were necessary because the first version of the MAX method only provides positive and negative cards for evaluating UX. Furthermore, in order to motivate the users to describe specific missing cards for depicting their opinion towards the evaluated application, we asked the following question: "If I gave you a blank card to express any emotion/feeling you think is missing, would you suggest any?" As a result, one of the subjects indicated the "impatient" card.

In general, the users indicated that they enjoyed employing MAX to describe their emotions. However, we still needed to improve the MAX method, based on the suggestions by the users. Regarding the cards, through our observations we noticed that the "empathic" card was not easy to understand and needed to be removed, as the users did not understand how they could feel empathy with an application. Also, we added the "impatient" card in the Emotion category and the "I gave up the task" in the Ease to Use category due to the users' suggestions. Furthermore, since all users reported that there were too many cards, we reduced the scale from four to three intensity levels ending up with 75 cards (17 less cards than in the first version). Fig. 6 shows the changes over the second version (v2) of the MAX cards.

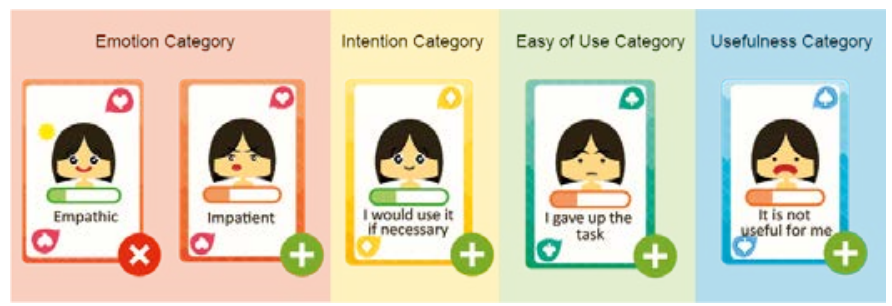

Figure 6. Changes over the MAX method (v2)

Regarding neutral cards, we chose not to add cards such as "I did not feel anything" or "Indifferent", as users who choose these items do not provide information regarding the side to which they are inclined (either positive or negative UX).
Furthermore, regarding the board, we removed the slots. Although we intended to encourage users to choose two or more cards, the slots made users select only two cards. Therefore, besides providing a larger space for selecting the cards in each category on the board, we also reduced the size of the cards, so two or more cards could be selected. The new size of the cards was also motivated by ergonomic principals to facilitate its use by both evaluators and users. We also made design changes in the cards to make them more minimalistic.

\section{CONCLUSIONS AND FUTURE WORK}

This paper presented MAX, which intends to be an easy to use method for both evaluators and users. Since the board guides users through the evaluation process, the evaluators do not need to be experts, making this method suitable for software engineers willing to gain insight in terms of UX. Also, by providing cards and a board that are intuitive and informal, we aimed at making users feel comfortable so they would not feel that the evaluation was a chore, but a pleasant and informal activity to identify their needs.

During the pilot study, the MAX method was perceived as a useful tool that can easily and quickly capture the overall opinion of the users regarding the evaluated artifact. As the EmoCards [17], by providing different cards, the MAX method allows users to think of different possibilities, while evaluating further aspects such as ease of use, usefulness and intention to use. Also, when compared to scales, users can feel free to complement the reasons that made them choose a specific card, providing evaluators with information on UX problems that need to be corrected. Moreover, the MAX method allows evaluators to interpret the emotion/expression in the drawings more easily, as it provides labels allowing the understanding of the cards meanings.

One of the limitations of our study was the small sample size employed. However, even with the small sample the results from this pilot study allowed us to test the applicability of the MAX method with positive results. Also, as the subjects who participated in the study were real end-users, their problems when employing the method could affect future applications of the method. Therefore, even though we could wait to refine the method after further studies, we decided to include the changes described in section IV so we can test their impact in the future applications of the MAX method.

Another limitation is that this initial evaluation was performed from the point of view of users. Since the evaluator was one of the authors of the technique, the application process could have been easier, affecting the overall opinion of the subjects. Therefore, we still need to carry out another evaluation from the point of view of software engineers, to verify what is needed to facilitate the use of the MAX method and the analysis of the results from the evaluation. Also, we need to verify if the opinion of users towards MAX remains the same, when being applied by software engineers. However, we can argue that an initial evaluation from the point of view of users is necessary before carrying out evaluations in industry, which are more expensive.

There could have also been a threat to the validity of our results in terms of the evaluated system and its 
representativeness. Although a Web application of a telecommunications company may not be representative of all types of applications (i.e. Web, mobile, desktop, others), it is still a real application which can yield different experiences. This study showed that MAX managed to capture such difference, by presenting opposing experiences from different users. However, we still need to verify the suitability of using MAX in evaluating other types of artifacts, such as prototypes, in which only part of the application has been developed. Finally, one last limitation is the instrument and measures applied in this study for assessing the users' opinion towards the MAX method. However, we believe that applying questionnaires was more suitable than applying interviews due to time constrains. Furthermore, evaluating if a UX technique is easy to use and to apply from the point of view of users is relevant in order to meet users' needs and make them feel comfortable during the evaluation process [7][20].

As future work we intend to carry out further empirical evaluations with the improved version of the MAX method in industrial and not controlled scenarios with more subjects/evaluators. Such evaluations aim at generalizing our results to further contexts and at evaluating if the current changes have an impact in the results of the UX evaluation. We also intend to carry out further comparative studies with the method described in Section II and other UX evaluation methods, to identify the situations in which MAX is more suitable to be applied. Among the improvement opportunities, we intend to develop other avatars for the MAX cards (i.e. with different genders and races) and evaluate their impact on the users' choice through comparative studies. Furthermore, while we intended to understand the degree of impact of an item from the MAX cards by its intensity, we noticed that the number of cards could have a cognitive overload over the users. Thus, we will check whether the scale also impacts the description of the UX through more empirical studies, and if needed, we will reduce it. Finally, we intend to develop a ready to use version of MAX, containing the instructions, cards and board. Such version will be useful for practitioners aiming at employing MAX at work or in the field.

\section{ACKNOWLEDGMENTS}

We thank CNPq for the scholarship granted to the second author of this paper and for its financial support through process $n^{\circ} 460627 / 2014-7$. Also, we thank the financial support granted by FAPEAM through processes $\mathrm{n}^{\circ}$ : 01135/2011; 062.00146/2012; 062.00600/2014; 062.00578/2014; and PAPE 004/2015. Finally, we would like to acknowledge the support granted by FPF Tech.

\section{REFERENCES}

[1] J. Bargas-Avila, K. Hornbæk, “Old wine in new bottles or novel challenges: a critical analysis of empirical studies of user experience”, In Procceedings of the SIGCHI Conference on Human Factors in Computing Systems, pp. 2689-2698, 2011.

[2] A. Fernandez, E. Insfran and S. Abrahao, "Usability evaluation methods for the Web: A systematic mapping study”, Information and Software Technology, 53(8), pp. 789-817, 2011.
[3] M. Hassenzahl, S. Diefenbach, A. Göritz, "Needs, affect, and interactive products-Facets of user experience”, Interacting with computers, pp. 353-362, 2010.

[4] International Standardization Organization, ISO 9241-210: Ergonomics of human system interaction, Part 210: Humancentred design for interactive systems, 2010.

[5] E. Law, V. Roto, M. Hassenzahl, A. Vermeeren, J. Kort, "Understanding, scoping and defining user experience: a survey approach”, Proceedings of Human Factors in Computing Systems conference, CHI’09, pp. 719-728, 2009.

[6] M. Tähti, L. Arhippainen, "A Proposal of collecting Emotions and Experiences”, In Interactive Experiences in HCI, Volume 2, pp. 195-198, 2004.

[7] A. Vermeeren, L. Law, V. Roto, M. Obrist, J. Hoonhout, K. Väänänen-Vainio-Mattila, "User experience evaluation methods: current state and development needs", In Proceedings of the 6th Nordic Conference on HCI, pp. 521-530, 2010.

[8] R. Miles, J. Greensmith, H. Schnadelbach, J. Garibaldi, "Towards a method of identifying the causes of poor user experience on websites”, In Computational Intelligence, UKCI, pp. 258-265, 2013.

[9] J. Preece, Y. Rogers, H. Sharp, "Interaction design: Beyond Human-Computer Interaction”, Wiley New York, 2002.

[10] V. Roto, "User Experience from Product Creation Perspective. Towards a UX Manifesto workshop”, In Conjunction with HCI, pp. 11-15, 2007.

[11] M. Hassenzahl, "User Experience - a Research Agenda”, In: Behaviour and Information Technology, 25(2), pp. 91-97, 2006.

[12] A. Moreno, A. Seffah, R. Capilla, M. Sanchez-Segura, M, "HCI Practices for Building Usable Software”, Computer, pp. 100102, 2013.

[13] L. Yong, "User experience evaluation methods for mobile devices”, In Proceedings III International Conference on Innovative Computing Technology, pp. 281-286, 2013.

[14] P. Lang, "Behavioral Treatment and Bio-behavioral Assessment Computer Applications”, In Technology in Mental Health Care Delivery Systems, pp. 119-137, 1980.

[15] V. Venkatesh, M. Morris, G. Davis, F. Davis, "User acceptance of information technology: Toward a unified view”, MIS quarterly, pp. 425-478, 2003.

[16] M. Hassenzahl, M. Burmester, F. Koller, “AttrakDiff: Ein Fragebogen zur Messung wahrgenommener hedonischer und pragmatischer Qualität”, In Mensch \& Computer 2003, pp. 187196, 2003.

[17] P. Desmet, C. Overbeeke, S. Tax, "Designing Products with Added Emotional Value: Development and Application of an Approach for Research through Design”, The Design Journal, pp. 32-47, 2001.

[18] M. Hassenzahl, "The thing and I: understanding the relationship between user and product”, In Funology, Springer Netherlands, pp. 31-42, 2005.

[19] R. Plutchik, "The Nature of Emotions Human emotions have deep evolutionary roots, a fact that may explain their complexity and provide tools for clinical practice”, American Scientist, 89(4), pp. 344-350, 2001.

[20] M. Isomursu, M. Tähti, S. Väinämö, K. Kuutti, "Experimental evaluation of five methods for collecting emotions in field settings with mobile applications", International Journal of Human-Computer Studies, 65(4), pp. 404-418, 2007. 